\title{
Estudo Comparativo de Polimerização de Propileno com Diferentes Catalisadores Metalocênicos Através de um Planejamento de Experimentos
}

\author{
Maria de Fátima V. Marques, Mariana Poloponsky, Érica G. Chaves \\ Instituto de Macromoléculas Professora Eloisa Mano, UFRJ
}

Resumo: Neste trabalho, o desempenho dos catalisadores $\mathrm{SiMe}_{2}(\mathrm{Ind})_{2} \mathrm{ZrCl}_{2}, \mathrm{Et}(\mathrm{Ind})_{2} \mathrm{ZrCl}_{2}, \mathrm{SiMe}_{2}(\mathrm{Ind})_{2} \mathrm{HfCl}_{2} \mathrm{e}$ $\mathrm{Et}(\mathrm{Ind})_{2} \mathrm{HfCl}_{2}$ na polimerização de propileno usando MAO como cocatalisador empregando-se um Planejamento estatístico de experimentos foi avaliado. As polimerizações foram realizadas em diferentes temperaturas e razões molares alumínio/metal de transição. O efeito destas variáveis na atividade de cada catalisador e nas características do polipropileno obtido foi melhor investigado utilizando-se os modelos propostos para cada variável. Foram observadas influências significativas das condições experimentais principalmente na atividade catalítica, bem como no peso molecular ponderal médio, temperatura de fusão e percentagem de isotaticidade dos polímeros obtidos. A partir dos modelos propostos através do tratamento estatístico realizado pode-se observar que os catalisadores a base de zircônio são os de maior atividade catalítica, enquanto que os hafnocenos produzem polipropileno com peso molecular mais elevado. Os complexos com ponte dimetil-silânica produziram polipropileno com maior peso molecular, estereorregularidade e maior temperatura de fusão do que os similares com ponte etilidênica.

Palavras-chave: Ansa-metaloceno, polipropileno, metal de transição.

\section{Comparative Study of Propylene Polymerization with Different Metallocene Catalysts Using a Statistic Experimental Planning Model}

\begin{abstract}
In this work, the performance of the catalysts $\mathrm{SiMe}_{2}(\mathrm{Ind})_{2} \mathrm{ZrCl}_{2}, \mathrm{Et}(\mathrm{Ind})_{2} \mathrm{ZrCl}_{2}, \mathrm{SiMe}_{2}(\mathrm{Ind})_{2} \mathrm{HfCl}_{2} \mathrm{e}$ $\mathrm{Et}(\mathrm{Ind})_{2} \mathrm{HfCl}_{2}$ on propylene polymerization using $\mathrm{MAO}$ as cocatalyst and employing a Statistic Experimental Planning Model was evaluated. The polymerizations were carried out at different temperatures and aluminum/ transition metal molar ratios. The effect of these variables on the catalyst activity and on the polymer characteristics was investigated using the proposed models for each variable. A significant influence was observed of the experimental conditions on the catalyst activity in particular, but also on the weight-average molecular weight, melting point and isotacticity of the polypropylenes produced; the statistical analysis with the proposed models indicated higher catalyst activity for the zirconocenes, while the hafnocenes produced polypropylene with higher molecular weight. The complexes with dimethylsilane bridge produced polypropylene with higher molecular weight, stereoregularity and higher melting temperature in comparison with the corresponding polymers using the ethylidene bridge.
\end{abstract}

Keywords: Ansa-metallocene, polypropylene, transition metal.

\section{Introdução}

Sistemas catalíticos metalocênicos têm sido extensivamente utilizados para polimerizar olefinas com altas atividades. Estes sistemas proporcionam um excelente controle da microestrutura do polímero, distribuição de peso molecular e incorporação de comonômero, resultando em novos materiais poliolefínicos disponíveis em escala comercial. A utilização destes catalisadores no processo de polimerização de olefinas é considerada uma etapa revolucionária na história dos polímeros ${ }^{[1-3]}$.

Autor para correspondência: Maria de Fátima V. Marques, Instituto de Macromoléculas Professora Eloisa Mano, UFRJ, Caixa Postal 68525, CEP: 21945-970, Rio de Janeiro, RJ.E-mail:fmarques@ima.ufrj.br 
Os primeiros sistemas catalíticos metalocênicos estudados, do tipo $\mathrm{Cp}_{2} \mathrm{TiCl}_{2} / \mathrm{AlR}_{\mathrm{x}} \mathrm{Cl}_{3-\mathrm{x}}$ mostravam baixas atividades para polimerização de etileno e não polimerizavam propileno. Assim, o sucesso da aplicação de metalocenos na polimerização de olefinas começou com duas intervenções: o uso do metilaluminoxano, MAO, como cocatalisador e o uso de pontes entre os ligantes na estrutura dos catalisadores (ansa-complexos para polimerização de propileno), o que viabilizou a comercialização do polipropileno isotático a partir de 1995 (Fina Oil and Chemicals, Exxon Chemical e Hoechst, por exemplo). As resinas a base de propileno produzidas por catalisadores metalocênicos são de grande interesse das indústrias petroquímicas e de transformação. Estes materiais possuem algumas características peculiares, tais como polidispersão estreita, baixa cristalinidade e baixo teor de extraíveis, o que leva a diferenças significativas no processo de transformação e nas características dos produtos finais ${ }^{[4-8]}$. O MAO aumenta a atividade dos sistemas metalocênicos em várias ordens de grandeza, comparando-se com compostos organoalumínio mais convencionais, devido ao fato de prontamente formar a espécie catiônica ativa. A adição do substituinte que constitui a ponte tornou o complexo estereorrígido, fornecendo simetria $\mathrm{C}_{\mathrm{S}}$ ao complexo sindioespecífico e simetria $\mathrm{C}_{2}$ ao isoespecífico. As propriedades dos polímeros obtidos com estes catalisadores são fortemente dependentes das condições experimentais para sua síntese $\mathrm{e}^{[9-11]}$.

Metalocenos isoespecíficos como, por exemplo, o rac-Et(Ind) ${ }_{2} \mathrm{ZrCl}_{2}$, são estereorrígidos, já que a ponte evita a livre rotação em torno da ligação $\eta^{5}$ entre o ligante indenila e o metal de transição, e pertence ao grupo que possui simetria do tipo $\mathrm{C}_{2}$.

A Tabela 1 mostra uma coletânea de algumas estruturas dos complexos metalocênicos e informações sobre seu desempenho, amplamente conhecidos pelo estado da arte, nas sínteses de polietileno e polipropileno, em relação basicamente ao peso molecular e microestrutura das cadeias de polipropileno resultantes.

Considerando a importância do polipropileno e o potencial dos metalocenos no mercado mundial, o objetivo deste trabalho foi, utilizando um planejamento estatístico de experimentos, avaliar o desempenho dos catalisadores $\mathrm{SiMe}_{2}$ (Ind) ${ }_{2} \mathrm{HfCl}_{2}$, Et(Ind) ${ }_{2} \mathrm{HfCl}_{2}$, $\mathrm{SiMe}_{2}(\mathrm{Ind})_{2} \mathrm{ZrCl}_{2}$ e Et(Ind) ${ }_{2} \mathrm{ZrCl}_{2}$ na polimerização de propileno, estudando a influência do tipo de ponte entre os ligantes e do metal de transição na atividade e nas características dos polímeros obtidos, apesar desses compostos terem sido utilizados largamente na literatura ${ }^{[2]}$.

\section{Experimental}

\section{Materiais}

Os experimentos para preparação de solução de catalisador e para polimerização foram realizados sob atmosfera de nitrogênio utilizando-se a técnica de Schlenk. Solução de MAO $10 \%$ em tolueno foi gentilmente cedida pela WITCO Co. e utilizada sem posterior purificação. Propileno (grau de polimerização), fornecido pela POLIBRASIL S.A. e nitrogênio (White Martins) foram tratados passando por coluna de peneira molecular $3 \AA$ e catalisador de cobre. Tolueno foi destilado na presença de sódio/benzofenona sob nitrogênio.

\section{Síntese dos catalisadores}

Os complexos $\mathrm{SiMe}_{2}$ (Ind) ${ }_{2} \mathrm{ZrCl}_{2}$ e Et(Ind) ${ }_{2} \mathrm{ZrCl}_{2}$ foram sintetizados de acordo com técnicas convencionais, conforme descrito na literatura ${ }^{[12,13]}$ enquanto que os complexos $\mathrm{SiMe}_{2}(\mathrm{Ind})_{2} \mathrm{HfCl}_{2} \mathrm{e} \mathrm{Et}(\mathrm{Ind})_{2} \mathrm{HfCl}_{2}$ foram gentilmente cedidos pela Witco Co.

\section{Polimerização}

As reações para a produção de polipropileno foram realizadas em autoclave de vidro Buchiglassuster BEP 280, de $500 \mathrm{~mL}$, acoplado com medidor de vazão mássica (modelo 5850D- Brooks Inst.) com escala de até $1000 \mathrm{NmL} / \mathrm{min}$ de monômero. O metaloceno e 50\% do MAO utilizado foram postos em pré-contato em tolueno, por 10 minutos e então adicionados à mistura monômero/solvente/MAO. Após uma hora, a purga foi aberta e a reação terminada com solução de $\mathrm{HCl} 5 \%$ em etanol. O polímero foi lavado e filtrado com água destilada e etanol até pH neutro, e então seco em estufa a vácuo a $60^{\circ} \mathrm{C}$ até peso constante.

Foi realizado o tratamento cinético dos dados fornecidos pelo equipamento, obtendo-se os perfis de velocidade, Rp (mol/l.s) versus tempo (min), para cada sistema catalítico em todas as condições experimentais.

A concentração de monômero no meio reacional (para 2 bar) foi calculada de acordo com a equação ${ }^{[14]}$ :

$$
\mathrm{X}_{\mathrm{C} 3 \mathrm{H} 6}=0.354-\left(6.75 \times 10^{-3}\right) \mathrm{T}+\left(3.66 \times 10^{-5}\right) \mathrm{T}^{2}
$$

Foram realizados testes de polimerização para os 
Tabela 1. Estruturas metalocênicas determinam a arquitetura do polímero

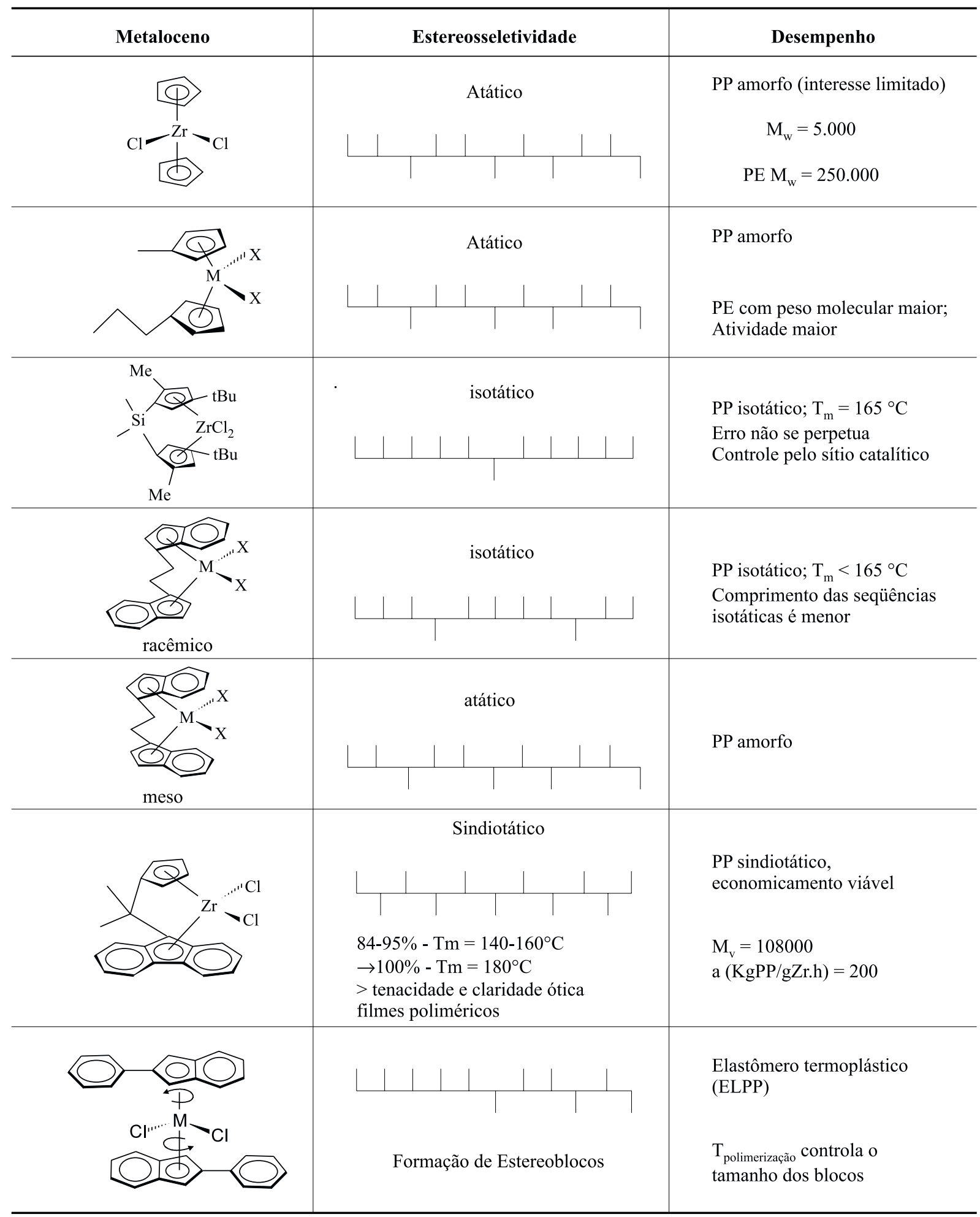

quatro complexos catalíticos em diferentes temperaturas de reação. Para os hafnocenos, que são mais estáveis à temperaturas mais elevadas, a $25^{\circ} \mathrm{C}$ não foi produzido polipropileno suficiente (baixo rendimen- to) para que os mesmos fossem analisados. Desta forma, os valores de temperatura de polimerização para estes catalisadores foram distintos dos zirconocenos. 


\section{Caracterização dos polímeros}

O peso molecular e a polidispersão dos polímeros foram determinados por Cromatografia de Exclusão por Tamanho (SEC) em aparelho Waters $150 \mathrm{CV}$, a $135^{\circ} \mathrm{C}$, utilizando $1,2,4$ triclorobenzeno como eluente, e com curva de calibração preparada a partir de padrões de poliestireno com polidispersão estreita.

Temperatura de fusão e grau de cristalinidade foram determinados por Calorimetria Diferencial de Varredura, em aparelho DSC 7 (Perkin Elmer), com taxa de aquecimento de $20^{\circ} \mathrm{C} / \mathrm{min}$, numa faixa de temperatura de $30-220^{\circ} \mathrm{C}$, em atmosfera de nitrogênio. O ciclo de aquecimento foi realizado duas vezes, mas somente os resultados da segunda corrida foram reportados.

Os valores de percentagem de isotaticidade (\% mmmm) dos polímeros sintetizados com o catalisador $\mathrm{Et}(\mathrm{Ind}){ }_{2} \mathrm{ZrCl}_{2}$ foram obtidos por Ressonância Magnética Nuclear $-{ }^{13} \mathrm{C}-\mathrm{NMR}$. Os espectros foram processados em Espectrômetro Varian modelo Mercury, operando a $300 \mathrm{MHz}$, com probe à $90^{\circ} \mathrm{C}$. As amostras foram preparadas dissolvendo-se o polímero em 1,2,4 triclorobenzeno e benzeno deuterado. Os resultados foram calculados com base na integração das áreas dos sinais correspondentes as pentades assinaladas na região de metila ${ }^{[3]}$.

Foi construída uma curva de calibração (Figura 1) com os valores de temperatura de fusão (Tm) e com os valores de percentagem de isotaticidade $(\% \mathrm{mmmm})$ calculados por 13C-NMR dos polímeros sintetizados com o catalisador $\mathrm{Et}(\mathrm{Ind})_{2} \mathrm{ZrCl}_{2}$. Desta forma, os valores de \%mmmm para os polímeros restantes foram calculados a partir da equação da curva de calibração construída. Assim, somente foi feito o tratamento estatístico para os polímeros obtidos com o catalisador $\mathrm{Et}(\mathrm{Ind})_{2} \mathrm{ZrCl}_{2}$.

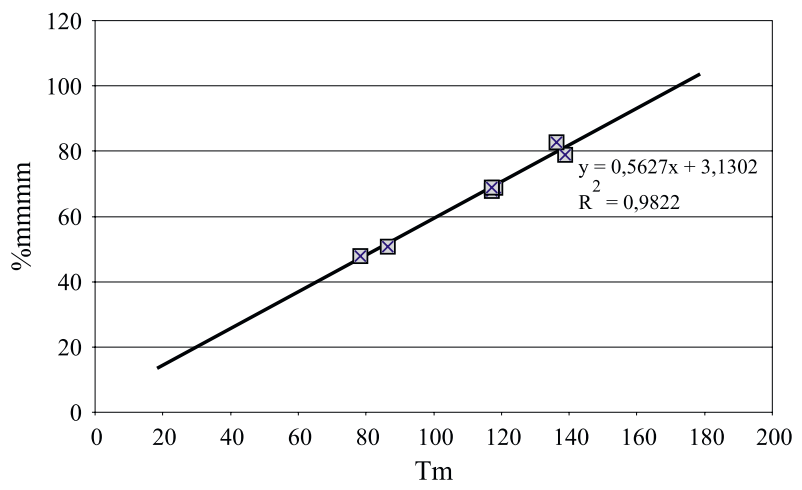

Figura 1. Relação entre a temperatura de fusão (Tm) e percentagem de isotaticidade $(\% \mathrm{mmmm})$
Tabela 2. Valores reais e codificados das variáveis estudadas

\begin{tabular}{|c|c|c|c|c|}
\hline & & \multicolumn{3}{|c|}{ níveis } \\
\hline catalisador & variável & -1 & $\mathbf{0}$ & +1 \\
\hline $\mathrm{SiMe}_{2}(\text { Ind })_{2} \mathrm{ZrCl}_{2}$ & $\begin{array}{l}\mathrm{X} 1 \text { - temperatura de } \\
\text { polimerização }\left({ }^{\circ} \mathrm{C}\right)\end{array}$ & 25 & 50 & 75 \\
\hline $\mathrm{Et}(\mathrm{Ind})_{2} \mathrm{ZrCl}_{2}$ & X2- razão $[\mathrm{Al}] /[\mathrm{Zr}]$ & 500 & 1250 & 2000 \\
\hline $\operatorname{SiMe}_{2}(\text { Ind })_{2} \mathrm{HfCl}_{2}$ & $\begin{array}{l}\mathrm{X} 1 \text { - temperatura de } \\
\text { polimerização }\left({ }^{\circ} \mathrm{C}\right)\end{array}$ & 40 & 60 & 80 \\
\hline $\mathrm{Et}(\mathrm{Ind})_{2} \mathrm{HfCl}_{2}$ & X2- razão $[\mathrm{Al}] /[\mathrm{Zr}]$ & 500 & 1250 & 2000 \\
\hline
\end{tabular}

\section{Análise Estatística}

Para cada catalisador, foi realizado um plano experimental, variando-se as condições reacionais. Assim, pôde-se realizar um estudo estatístico comparativo entre os catalisadores. Os experimentos envolveram um plano fatorial de dois níveis com diferenças uniformes nos parâmetros ${ }^{[15]}$. Os planos foram conduzidos segundo o planejamento de experimentos mostrados na Tabela 2. As variáveis foram temperatura de polimerização (X1) e razão molar alumínio/metal de transição (X2).

O planejamento de experimentos permitiu o cálculo de equações empíricas para cada uma das variáveis dependentes (atividade catalítica, Y1; temperatura de fusão, Y2; peso molecular, Y3; polidispersão, Y4; e grau de isotaticidade, Y5). O coeficiente de correlação R também foi calculado. Os planos consistem de sete corridas com três réplicas no ponto central.

Para todas as análises, foi utilizado o Software Statistica for Windows, versão 4.3.

\section{Resultados e Discussão}

Os resultados experimentais obtidos para as polimerizações de propileno com os catalisadores $\mathrm{SiMe}_{2}(\text { Ind })_{2} \mathrm{ZrCl}_{2}, \mathrm{Et}(\mathrm{Ind})_{2} \mathrm{ZrCl}_{2}, \mathrm{SiMe}_{2}(\mathrm{Ind})_{2} \mathrm{HfCl}_{2}$ e Et(Ind) ${ }_{2} \mathrm{HfCl}_{2}$ são mostrados nas Tabelas 3, 4, 5 e 6 , respectivamente. A partir destes resultados experimentais foram propostos modelos para cada variável, apresentados na Tabela 7.

A avaliação do significado das variáveis independentes nas diversas respostas estudadas foi feita com base nos valores dos parâmetros (constantes que multiplicam cada variável independente, X1 e X2) estimados pelo Software para cada equação do modelo. 
Influência das Variáveis Independentes na

Polimerização de Propileno com catalisador

SiMe $_{2}$ (Ind) ${ }_{2} \mathrm{ZrCl}_{2^{\prime}}$ (Tabela 3)

\section{Atividade Catalítica (Y1)}

Através da equação do modelo, obtida dos resultados experimentais mostrados na Tabela 3 pode-se observar que dentro da faixa estudada, a temperatura (X1) é a variável que mais influencia a atividade do catalisador $\mathrm{SiMe}_{2}$ (Ind) ${ }_{2} \mathrm{ZrCl}_{2}$. A velocidade de polimerização é proporcional à temperatura, o que aumenta a atividade dentro da faixa estudada. Por outro lado, o aumento da temperatura do meio reacional causa a diminuição da concentração de monômero dissolvido, à pressão constante. Em temperaturas altas, o segundo efeito é bastante acentuado, provocando queda da atividade. Em relação à razão $\mathrm{A} 1 / \mathrm{Zr}$ (X2), observou-se que existe uma razão ótima, onde a atividade catalítica é máxima (ver Figura 2a).

\section{Temperatura de Fusão (Y2)}

A temperatura reacional (X1) influencia mais diretamente a temperatura de fusão do polipropileno para este catalisador. A diminuição da Tm com o aumento da temperatura está diretamente relacionada com a influência desta variável independente no peso molecular e na isotaticidade do polímero, conforme será observado mais adiante.

\section{Peso molecular (Y3)}

Com relação ao peso molecular, este diminui principalmente com a temperatura de polimerização (X1).

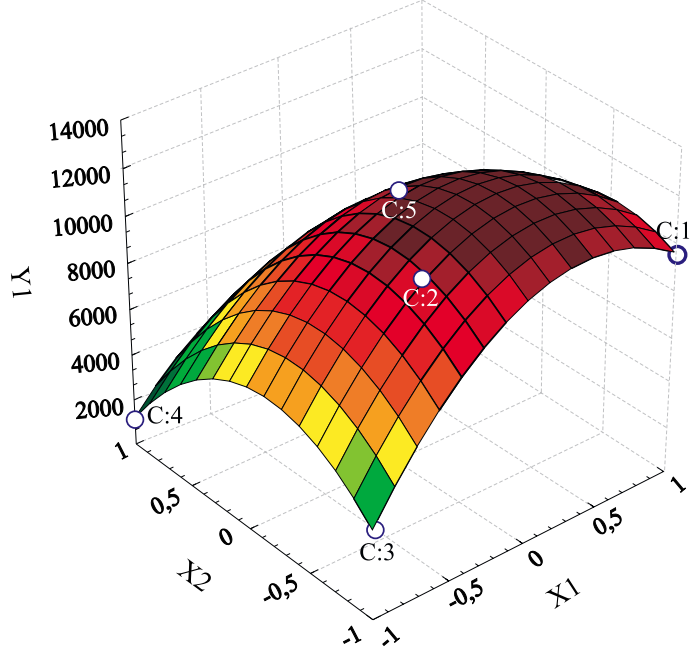

(a) atividade catalítica $(\mathrm{kg} /[\mathrm{Me}] \mathrm{h})$

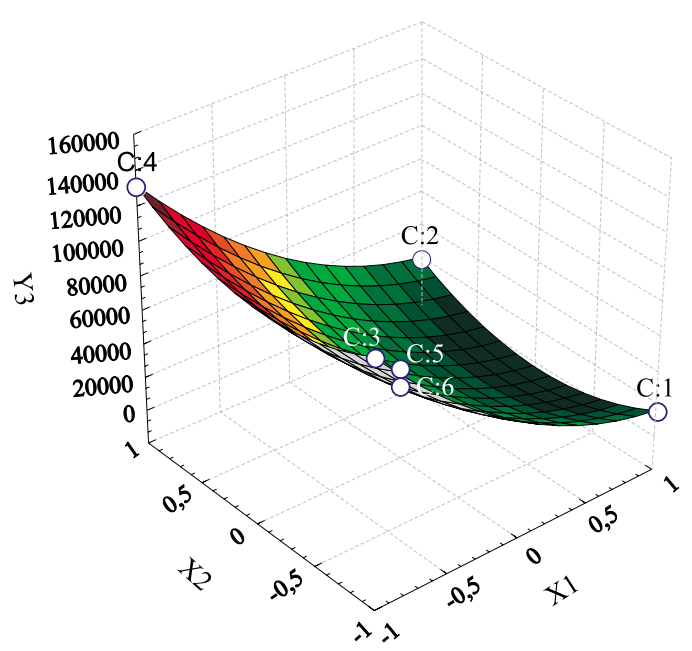

(b) peso molecular ponderal médio

Figura 2. Influência da temperatura de polimerização e da razão $\mathrm{Al} / \mathrm{Zr}$ nas variáveis dependentes utilizando o sistema catalítico $\mathrm{SiMe}_{2}(\mathrm{Ind})_{2} \mathrm{ZrCl}_{2} / \mathrm{MAO}$

Tabela 3. Polimerizações de propileno com o sistema catalítico $\mathrm{SiMe}_{2}(\mathrm{Ind})_{2} \mathrm{ZrCl}_{2} / \mathrm{MAO}$

\begin{tabular}{|c|c|c|c|c|c|c|c|c|c|}
\hline $\mathbf{N}^{\mathbf{o}}$ & $\begin{array}{c}\text { Tp }\left({ }^{\circ} \mathbf{C}\right) \\
\text { X1 }\end{array}$ & $\begin{array}{c}{[\mathrm{Al}] /[\mathrm{Zr}]} \\
\mathrm{X} 2\end{array}$ & $\begin{array}{c}\mathrm{a} \\
(\mathrm{KgP} /[\mathrm{Me}] \mathrm{h}) \\
\mathrm{Y} 1\end{array}$ & $\begin{array}{c}\mathrm{a}^{*} \\
(\mathrm{KgP} /[\mathrm{Me}][\text { Prop }] \mathrm{h})\end{array}$ & $\begin{array}{c}\text { Tm } \\
\left({ }^{\circ} \mathbf{C}\right) \\
\text { Y2 }\end{array}$ & $\begin{array}{l}\mathrm{Xc} \\
(\%)\end{array}$ & $\begin{array}{c}\text { Mw } \\
\text { x10 } \\
\text { Y3 }\end{array}$ & $\begin{array}{c}\text { Mw/Mn } \\
\text { Y4 }\end{array}$ & $\begin{array}{c}\text { mmmm } \\
(\%) \\
\text { Y5 }\end{array}$ \\
\hline 1 & 25 & 500 & 3900 & 1579 & 147 & 50 & 12,3 & 1,8 & 80 \\
\hline 2 & 25 & 2000 & 3900 & 1576 & 148 & 49 & 13,2 & 1,8 & 80 \\
\hline 3 & 75 & 500 & 6640 & 12528 & 99 & 16 & 1,5 & 2,8 & 53 \\
\hline 4 & 75 & 2000 & 13800 & 26037 & 123 & 36 & 1,9 & 2,6 & 66 \\
\hline 5 & 50 & 1250 & 9600 & 8421 & 135 & 45 & 3,6 & 2,8 & 73 \\
\hline 6 & 50 & 1250 & 11680 & 10245 & 123 & 39 & 2,2 & 2,9 & 66 \\
\hline 7 & 50 & 1250 & 11280 & 9895 & 133 & 39 & 3,3 & 2,6 & 72 \\
\hline
\end{tabular}

Tp- temperatura de polimerização; [Al]/[Zr]- razão entre a concentração de alumínio e a concentração de zircônio; a- atividade catalítica; a *atividade específica; Tm- temperatura de fusão; Xc- grau de cristalinidade; Mw- peso molecular ponderal médio, Mw/Mn- polidispersão; mmmmpercentagem de pentades isotáticas. 
O aumento da temperatura favorece as reações de transferência de cadeia com hidrogênio beta, diminuindo o peso molecular do polímero. Já o efeito da razão $\mathrm{Al} / \mathrm{Zr}(\mathrm{X} 2)$, na faixa estudada, é relativamente pequeno. A Figura 2 mostra o efeito das variáveis estudadas na atividade catalítica e no peso molecular do polipropileno obtido.

\section{Polidispersão (Y4)}

De acordo com o modelo, o aumento da temperatura (X1) aumenta a polidispersão, o que era um comportamento esperado devido ao favorecimento das reações de transferência de cadeia com hidrogênio beta, conforme já discutido anteriormente.

Os perfis de velocidade de polimerização versus tempo para este sistema catalítico são apresentados na Figura 3. À temperatura de $75^{\circ} \mathrm{C}$, em qualquer razão $\mathrm{Al} / \mathrm{Zr}$, o perfil é descendente, mostrando a instabilidade do sistema catalítico a temperaturas mais elevadas.

Influência das Variáveis Independentes na

Polimerização de Propileno com catalisador

Et(Ind) $\mathrm{ZrCl}_{2 \cdot}$ (Tabela 4)

\section{Atividade Catalítica (Y1)}

A equação do modelo permite observar que, dentro da faixa estudada, a temperatura de polimerização (X1) e a razão $\mathrm{Al} / \mathrm{Zr}$ (X2) influenciam de maneira aproximadamente equivalente na atividade do catalisador $\mathrm{Et}(\mathrm{Ind})_{2} \mathrm{ZrCl}_{2}$. A baixas temperaturas e razões $\mathrm{Al} / \mathrm{Zr}$, a atividade catalítica é mínima. Uma explicação para este fato é que provavelmente, no início da reação nestas condições, o número de sítios ativos é ainda pequeno, e vai aumentando lentamente com o tempo, apresentando um período de indução, o que pode ser observado no respectivo perfil de velocidade de polimerização apresentado posteriormente.

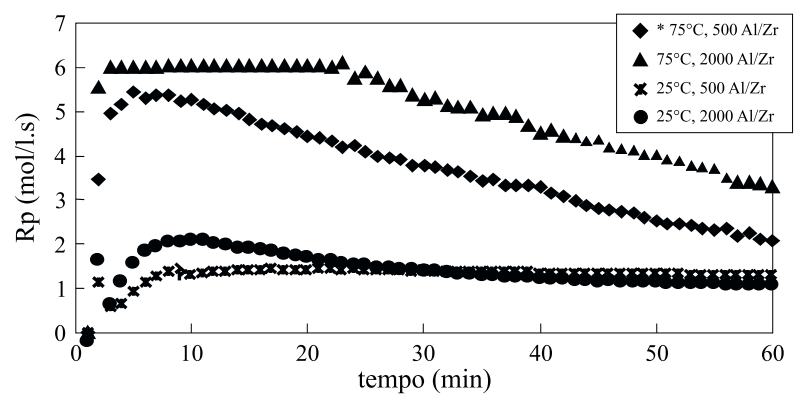

Figura 3. Perfil de velocidade de polimerização de propileno com o catalisador $\mathrm{SiMe}_{2}$ (Ind) $\mathrm{ZrCl}_{2}$

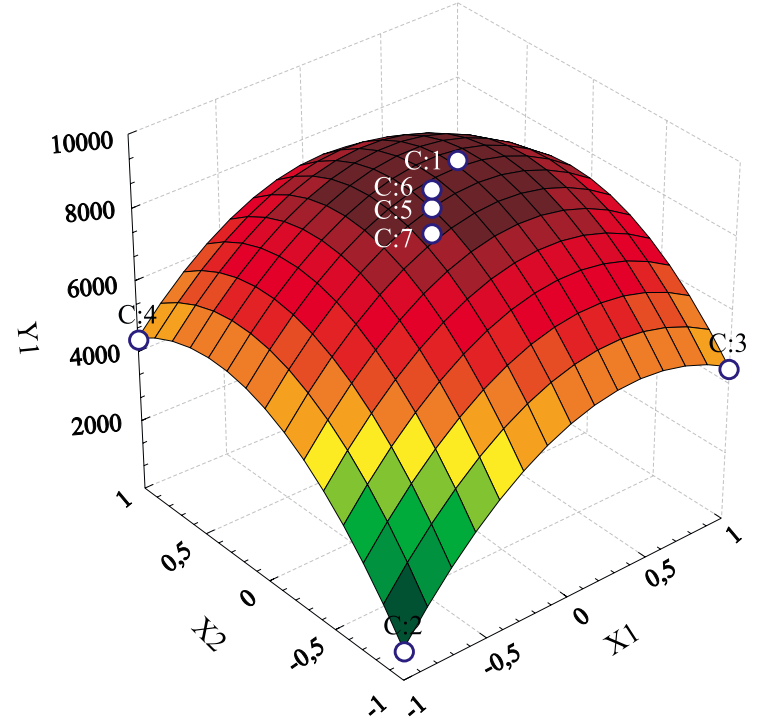

(a) atividade catalítica $(\mathrm{kg} /[\mathrm{Me}] \mathrm{h})$

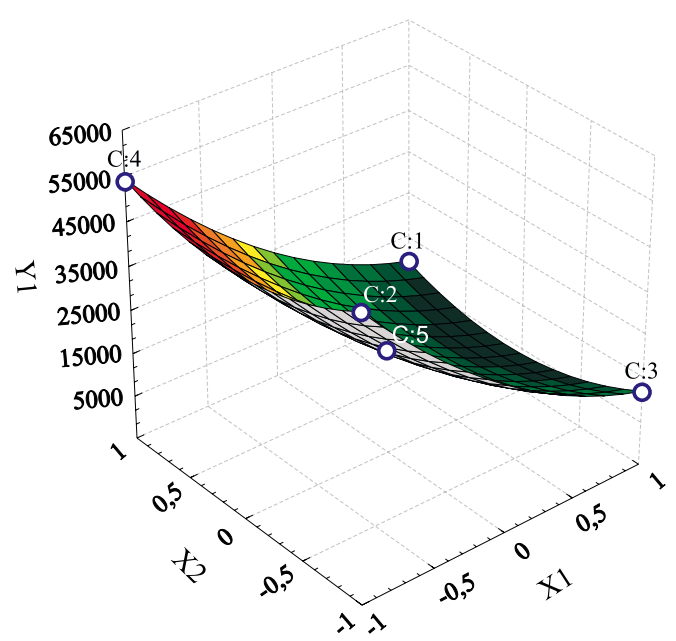

(b) peso molecular ponderal médio

Figura 4. Influência da temperatura de polimerização e da razão $\mathrm{Al} / \mathrm{Zr}$ nas variáveis dependentes utilizando o sistema catalítico $\mathrm{Et}(\mathrm{Ind}){ }_{2} \mathrm{ZrCl}_{2} / \mathrm{MAO}$

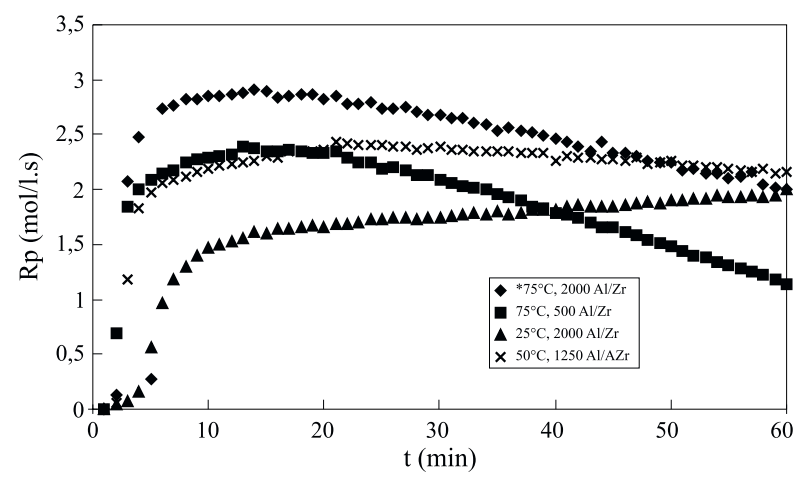

Figura 5. Perfil de velocidade de polimerização de propileno com o catalisador $\mathrm{Et}(\mathrm{Ind})_{2} \mathrm{ZrCl}_{2}$ 
Tabela 4. Polimerizações de propileno com o sistema catalítico Et(Ind) $\mathrm{ZrCl}_{2} / \mathrm{MAO}$

\begin{tabular}{|c|c|c|c|c|c|c|c|c|c|}
\hline $\mathbf{N}^{\mathbf{o}}$ & $\begin{array}{c}\mathbf{T p}\left({ }^{\circ} \mathbf{C}\right) \\
\mathbf{X 1}\end{array}$ & $\begin{array}{c}{[\mathrm{Al}] /[\mathrm{Zr}]} \\
\mathrm{X} 2\end{array}$ & $\begin{array}{c}\text { a } \\
(\mathrm{KgP} /[\mathrm{Me}] \mathrm{h}) \\
\text { Y1 }\end{array}$ & $\begin{array}{c}\mathbf{a}^{*} \\
\text { (KgP/[Me][Prop]h) } \\
\text { Y1 }\end{array}$ & $\begin{array}{c}\mathbf{T m} \\
\left({ }^{\circ} \mathbf{C}\right) \\
\mathbf{Y 2}\end{array}$ & $\begin{array}{c}\mathrm{Xc} \\
(\%) \\
\mathrm{Y3}\end{array}$ & $\begin{array}{c}\text { Mw } \\
\mathbf{x 1 0 ^ { 4 }} \\
\text { Y4 }\end{array}$ & $\begin{array}{c}\text { Mw/Mn } \\
\text { Y5 }\end{array}$ & $\begin{array}{c}\text { mmmm } \\
(\%) \\
\text { Y6 }\end{array}$ \\
\hline 1 & 25 & 500 & 900 & 364 & 138 & 39 & 6,3 & 2,4 & 79 \\
\hline 2 & 25 & 2000 & 4380 & 1773 & 136 & 42 & 5,4 & 2,2 & 83 \\
\hline 3 & 75 & 500 & 4400 & 8301 & 86 & —* & 1,2 & 2,1 & 51 \\
\hline 4 & 75 & 2000 & 5660 & 10679 & 78 & —* & 0,8 & 1,9 & 48 \\
\hline 5 & 50 & 1250 & 7780 & 6825 & 118 & 28 & 1,9 & 2,4 & 69 \\
\hline 6 & 50 & 125 & 8480 & 7438 & 117 & 26 & 1,9 & 2,4 & 68 \\
\hline 7 & 50 & 1250 & 8900 & 7807 & 117 & 29 & 2,0 & 2,4 & 69 \\
\hline
\end{tabular}

Tp- temperatura de polimerização; [Al]/[Zr]- razão entre a concentração de alumínio e a concentração de zircônio; a- atividade catalítica; a *atividade específica; Tm- temperatura de fusão; Xc- grau de cristalinidade; Mw- peso molecular ponderal médio, Mw/Mn- polidispersão; mmmmpercentagem de pentades isotáticas; * amorfo até $200^{\circ} \mathrm{C}$

Tabela 5. Resultados obtidos na polimerização de propileno com sistema catalítico $\mathrm{SiMe}_{2}(\mathrm{Ind})_{2} \mathrm{HfCl}_{2} / \mathrm{MAO}$

\begin{tabular}{|c|c|c|c|c|c|c|c|c|c|}
\hline $\mathbf{N}^{\mathbf{o}}$ & $\mathbf{T}\left({ }^{\circ} \mathbf{C}\right)$ & {$[\mathbf{A l}] /[\mathbf{Z r}]$} & $\begin{array}{c}\text { a } \\
\text { (Kg/molHf.h) } \\
\text { Y1 }\end{array}$ & $\begin{array}{c}\mathbf{a}^{*} \\
(\mathrm{Kg} /[\mathrm{Me}][\text { Prop }] . h)\end{array}$ & $\begin{array}{c}\text { Tm } \\
\left({ }^{\circ} \mathbf{C}\right) \\
\text { Y2 }\end{array}$ & $\begin{array}{l}\mathrm{Xc} \\
(\%)\end{array}$ & $\begin{array}{c}\text { Mw } \\
\text { x10 } \\
\text { Y3 }\end{array}$ & $\begin{array}{c}\text { Mw/Mn } \\
\text { Y4 }\end{array}$ & $\begin{array}{c}\text { mmmm } \\
(\%) \\
\text { Y5 }\end{array}$ \\
\hline 1 & 40 & 500 & 660 & 431 & 134 & 20 & 34,6 & 2,9 & 72 \\
\hline 2 & 40 & 2000 & 900 & 588 & 135 & 36 & 27,1 & 2,8 & 73 \\
\hline 3 & 80 & 500 & 1460 & 3042 & 125 & 16 & 6,2 & 1,9 & 67 \\
\hline 4 & 80 & 2000 & 1700 & 3542 & 127 & 17 & 3,9 & 1,9 & 68 \\
\hline 5 & 60 & 1250 & 1600 & 1928 & 134 & 41 & 10,4 & 2,2 & 72 \\
\hline 6 & 60 & 1250 & 1700 & 2048 & 134 & 36 & 11,7 & 2,2 & 72 \\
\hline 7 & 60 & 1250 & 1478 & 1781 & 137 & 38 & 11,5 & 2,2 & 74 \\
\hline
\end{tabular}

Tp- temperatura de polimerização; [Al]/[Hf]- razão entre a concentração de alumínio e a concentração de háfnio; a- atividade catalítica; a *atividade específica; Tm- temperatura de fusão; Xc- grau de cristalinidade; Mw- peso molecular ponderal médio, Mw/Mn- polidispersão; mmmmpercentagem de pentades isotáticas

Tabela 6. Resultados obtidos na polimerização de propileno com sistema catalítico Et(Ind) ${ }_{2} \mathrm{HfCl}_{2} / \mathrm{MAO}$

\begin{tabular}{|c|c|c|c|c|c|c|c|c|c|}
\hline $\mathbf{N}^{\mathbf{o}}$ & $\mathbf{T p}\left({ }^{\circ} \mathbf{C}\right)$ & {$[\mathrm{Al}] /[\mathrm{Zr}]$} & $\begin{array}{c}\mathbf{a} \\
(\mathrm{Kg} /[\mathrm{Me}] \mathrm{h}) \\
\text { Y1 }\end{array}$ & $\begin{array}{c}\mathrm{a}^{*} \\
(\mathrm{Kg} /[\mathrm{Me}][\mathrm{Prop}] \mathrm{h}) \\
\text { Y1 }\end{array}$ & $\begin{array}{l}\text { Tm } \\
\left({ }^{\circ} \mathbf{C}\right) \\
\text { Y2 }\end{array}$ & $\begin{array}{c}\mathrm{Xc} \\
(\%)\end{array}$ & $\begin{array}{c}\text { Mw } \\
\mathbf{x 1 0 ^ { 4 }} \\
\mathbf{Y 3}\end{array}$ & $\begin{array}{c}\text { Mw/Mn } \\
\text { Y4 }\end{array}$ & $\begin{array}{c}\text { mmmm } \\
(\%) \\
\text { Y5 }\end{array}$ \\
\hline 1 & 40 & 500 & 400 & 261 & 134 & 33 & 221000 & 1,83 & 72 \\
\hline 4 & 40 & 2000 & 1380 & 902 & 134 & 37 & 177000 & 1,85 & 72 \\
\hline 2 & 80 & 500 & 100 & 208 & 118 & 28 & 29000 & 2,78 & 63 \\
\hline 7 & 80 & 2000 & 1880 & 3916 & 117 & 28 & 20000 & 2,18 & 63 \\
\hline 3 & 60 & 1250 & 1500 & 1807 & 131 & 36 & 65000 & 1,60 & 70 \\
\hline 5 & 60 & 1250 & 2440 & 2940 & 128 & 34 & 57000 & 1,96 & 69 \\
\hline 6 & 60 & 1250 & 1780 & 2171 & 129 & 36 & 62000 & 1,77 & 69 \\
\hline
\end{tabular}

Tp- temperatura de polimerização; [Al]/[Hf]- razão entre a concentração de alumínio e a concentração de háfnio; a- atividade catalítica; a*atividade específica; Tm- temperatura de fusão; Xc- grau de cristalinidade; Mw- peso molecular ponderal médio, Mw/Mn- polidispersão; mmmmpercentagem de pentades isotáticas 


\section{Temperatura de Fusão (Y2)}

A temperatura de polimerização (X1) influencia negativamente a temperatura de fusão do polipropileno, assim como para o catalisador $\mathrm{SiMe}_{2}(\mathrm{Ind})_{2} \mathrm{ZrCl}_{2}$.

\section{Peso Molecular (Y3)}

Com relação ao peso molecular, de acordo com o modelo proposto, este diminui principalmente com a temperatura de polimerização (X1). Como explicado anteriormente, o aumento da temperatura favorece as reações de transferência de cadeia, diminuindo o peso molecular. O peso molecular também diminuiu com o teor de MAO, já que o aumento da concentração de alumínio leva às reações de transferência de cadeia com as moléculas de MAO. Os dois efeitos contribuem para a diminuição do peso molecular do polímero. A Figura 4 mostra a influência das variáveis estudadas na atividade e no peso molecular do polipropileno produzido.

\section{Polidispersão (Y4)}

De acordo com o modelo, o aumento da temperatura de polimerização $(\mathrm{X} 1)$ parece diminuir ligeiramente a polidispersão do polímero. Uma explicação para isto seria que, a maior temperatura, quase todos os sítios de polimerização já estariam ativos desde o início da reação, as cadeias poliméricas estariam então se iniciando ao mesmo tempo, provocando a queda da polidispersão.

\section{Isotaticidade (Y5)}

Pela equação do modelo, pode-se observar que a

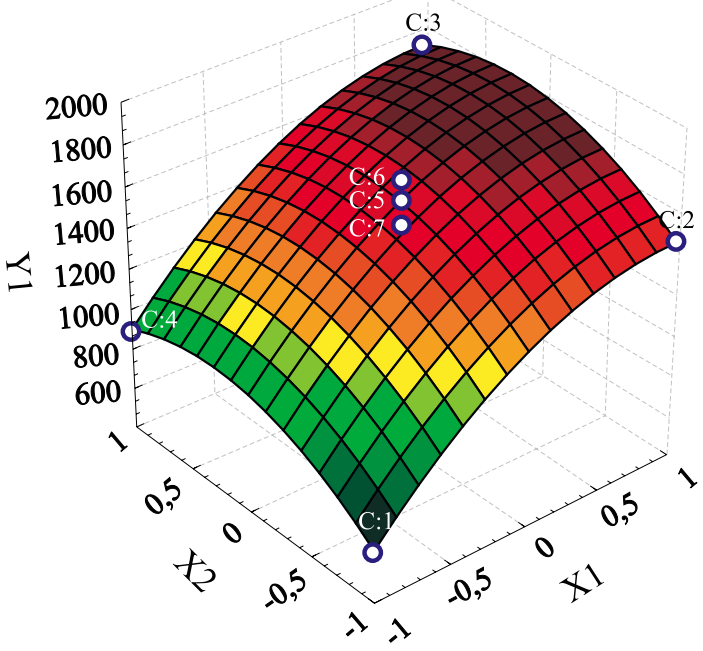

(a) atividade catalítica $(\mathrm{kg} /[\mathrm{Me}] \mathrm{h})$ temperatura de polimerização (X1) é a variável que mais influencia a isotaticidade do polipropileno sintetizado por este catalisador, na faixa estudada. Este comportamento já era esperado, considerando que o aumento da temperatura promove uma maior flexibilidade da molécula do complexo catalítico, aumentando também a energia do sistema. Isto favorece as inserções não específicas, diminuindo a isotaticidade dos polímeros obtidos.

Os perfis de velocidade de polimerização para o catalisador Et(Ind) ${ }_{2} \mathrm{ZrCl}_{2}$ são mostrados na Figura 5. A $75^{\circ} \mathrm{C}$ o perfil é descendente, especialmente a menor razão $\mathrm{Al} / \mathrm{Zr}$, mostrando o papel do $\mathrm{MAO}$, que estabiliza o sítio ativo. A $25^{\circ} \mathrm{C}$ a reação parece apresentar um período de indução.

Influência das Variáveis Independentes na Polimerização de Propileno com catalisador SiMe $_{2}$ (Ind) ${ }_{2} \mathrm{HfCl}_{2}$ (Tabela 5)

\section{Atividade Catalítica (Y1)}

Através da equação do modelo, pode-se observar que, dentro da faixa estudada, a temperatura de polimerização $(\mathrm{X} 1)$ foi a variável que mais influenciou na atividade do catalisador $\mathrm{SiMe}_{2}(\mathrm{Ind})_{2} \mathrm{HfCl}_{2}$. Aumentando-se a temperatura e a razão Al/Hf (X2), observou-se um aumento da atividade, que foi bem mais acentuado quando se considerou a diminuição da concentração de monômero no meio reacional com o aumento da temperatura (atividade específica). A velocidade de polimerização aumenta com o aumento da constante de velocidade de propagação e a ge-

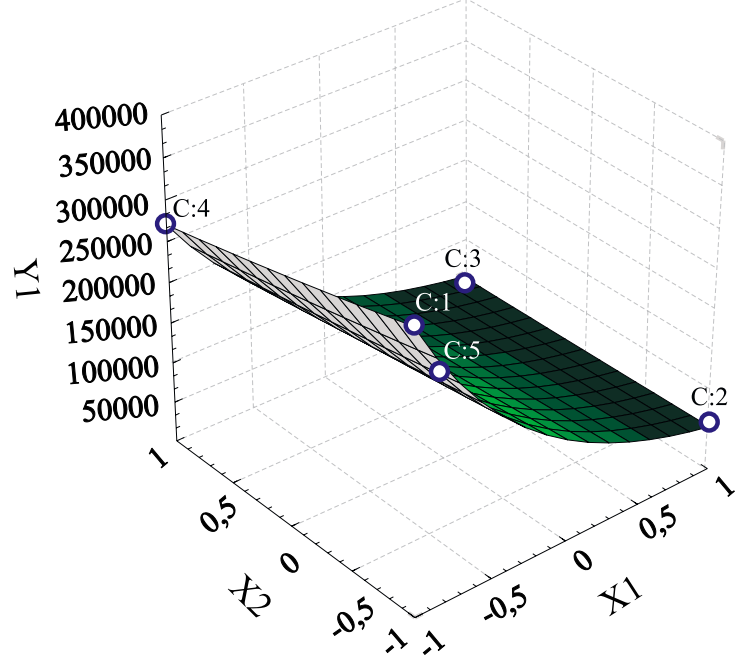

(b) peso molecular ponderal médio

Figura 6. Influência da temperatura de polimerização e da razão $\mathrm{Al} / \mathrm{Zr}$ nas variáveis dependentes utilizando o sistema catalítico $\mathrm{SiMe}_{2}(\mathrm{Ind})_{2} \mathrm{HfCl}_{2} / \mathrm{MAO}$ 
ração de centros ativos durante a reação. Esses dois fatores são favorecidos principalmente pelo aumento da temperatura e da razão $\mathrm{Al} / \mathrm{Hf}$, respectivamente.

\section{Temperatura de Fusão (Y2)}

De acordo com a equação do modelo, a temperatura de polimerização (X1) influenciou negativamente a temperatura de fusão do polipropileno obtido com o catalisador $\mathrm{SiMe}_{2}(\mathrm{Ind})_{2} \mathrm{HfCl}_{2}$. Isto provavelmente se deve ao fato de ter ocorrido diminuição da estereorregularidade do polímero, com a introdução de defeitos cristalinos, além da diminuição do peso molecular.

\section{Peso Molecular (Y3)}

Com relação ao peso molecular, este diminuiu exponencialmente com a razão $\mathrm{Al} / \mathrm{Hf}(\mathrm{X} 2)$ e, principalmente, com a temperatura de polimerização (X1). $\mathrm{O}$ aumento da temperatura favoreceu as reações de transferência de cadeia, conforme mencionado anteriormente. O aumento da concentração de alumínio levou às reações de transferência de cadeia com as moléculas do cocatalisador, diminuindo o peso molecular do polipropileno obtido. A Figura 6 mostra o efeito das variáveis estudadas na atividade catalítica e no peso molecular do polipropileno para este catalisador.

\section{Polidispersão (Y4)}

Curiosamente foi observado que a polidispersão estreitou levemente com o aumento da temperatura de reação (X1) no caso do catalisador com ponte de dimetilsilano. Pode-se compreender melhor esta observação através dos perfis de velocidade de polimerização versus tempo, apresentados na Figura 7. A polimerização realizada a $40^{\circ} \mathrm{C}$ e razão $\mathrm{Al} /$ Hf de 500 apresentou um período de indução, só

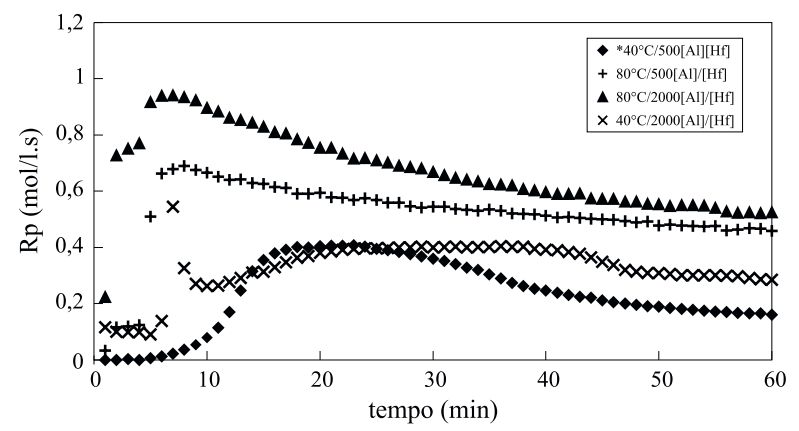

Figura 7. Perfil de velocidade de polimerização de propileno com o catalisador $\mathrm{SiMe}_{2}(\mathrm{Ind})_{2} \mathrm{HfCl}_{2}$ atingindo a velocidade de polimerização máxima após mais de 15 minutos de reação. Neste período ocorre o aumento lento do número de sítios ativos, e é devido a este efeito que a polidispersão do polímero se tornou alargada, próximo a 3. Com o aumento da razão $\mathrm{Al} / \mathrm{Hf}$ para 2000, não se observou período de indução tão acentuado, e a geração de sítios ativos se deu mais rapidamente, além da alta concentração de MAO estabilizar esses sítios por mais tempo, como mostra o perfil mais constante. Já a $80^{\circ} \mathrm{C}$, as velocidades de polimerização foram mais elevadas, atingindo valores máximos logo no início da reação, e decrescendo lentamente.

Influência das Variáveis Independentes na Polimerização de Propileno com catalisador Et(Ind) $\mathrm{HfCl}_{2}$ (Tabela 6)

\section{Atividade Catalítica (Y1)}

Ao contrário do que foi observado com o catalisador $\mathrm{SiMe}_{2}$ (Ind) ${ }_{2} \mathrm{HfCl}_{2}$, neste caso, a razão $\mathrm{Al} / \mathrm{Hf}(\mathrm{X} 2)$ é a variável que mais influencia na atividade catalítica (parâmetro de maior valor). Isto ocorreu provavelmente porque a $80^{\circ} \mathrm{C}$, a razão $\mathrm{Al} / \mathrm{Hf}$ de 500 foi insuficiente para estabilizar os sítios ativos gerados. O perfil de velocidade de polimerização será apresentado adiante.

\section{Temperatura de Fusão (Y2)}

A temperatura de polimerização (X1) é a variável mais significativa, influenciando negativamente a temperatura de fusão dos polipropilenos produzidos, conforme já discutido anteriormente.

\section{Peso Molecular (Y3)}

$\mathrm{O}$ aumento da temperatura de polimerização (X1) acarretou a diminuição do peso molecular do polímero, considerando que são favorecidas as reações de transferência de cadeia de hidrogênio beta. A influência da razão $\mathrm{Al} / \mathrm{Hf}(\mathrm{X} 2)$ também foi verificada, porém com menor intensidade, assim como foi observado para o catalisador $\mathrm{SiMe}_{2}$ (Ind) ${ }_{2} \mathrm{HfCl}_{2}$.

A Figura 8 mostra a influência das variáveis independentes na atividade e peso molecular dos polipropilenos produzidos a partir do sistema catalítico $\mathrm{Et}(\mathrm{Ind})_{2} \mathrm{HfCl}_{2} / \mathrm{MAO}$.

\section{Polidispersão (Y4)}

A polidispersão foi em geral estreita para a maio- 


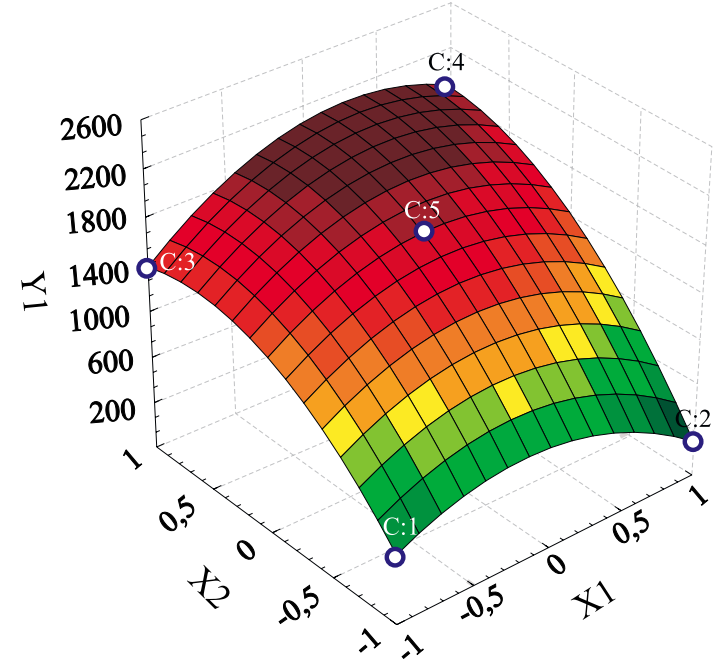

(a) atividade catalítica $(\mathrm{kg} /[\mathrm{Me}] \mathrm{h})$

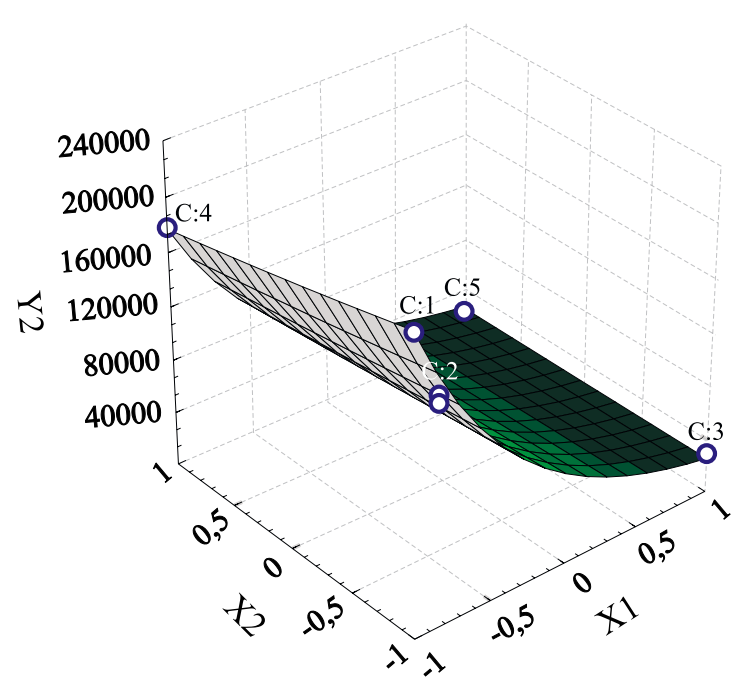

(b) peso molecular ponderal médio

Figura 8. Influência da temperatura de polimerização e da razão $\mathrm{Al} / \mathrm{Zr}$ nas variáveis dependentes utilizando o sistema catalítico $\mathrm{Et}(\mathrm{Ind})_{2} \mathrm{HfCl}_{2} / \mathrm{MAO}$

ria dos polipropilenos obtidos, alargando, porém, quando a temperatura da reação foi de $80^{\circ} \mathrm{C}$.

Os perfis de velocidade de polimerização versus tempo para este sistema catalítico são apresentados na Figura 9. As curvas não apresentaram período de indução. A curva de velocidade da polimerização realizada a $80^{\circ} \mathrm{C}$ e razão $\mathrm{Al} / \mathrm{Hf}=500$ é a única que apresentou desativação desde o início, com a velocidade decrescente, permanecendo baixa até o final da reação. Acredita-se que em razão molar $\mathrm{Al} / \mathrm{Hf}$ relativamente baixa, não foi possível estabilizar a maioria dos sítios ativos na temperatura de polimerização de $80^{\circ} \mathrm{C}$.

Dos resultados obtidos, comparando-se os desempenhos dos quatro catalisadores, é possível verificar que as atividades dos catalisadores com

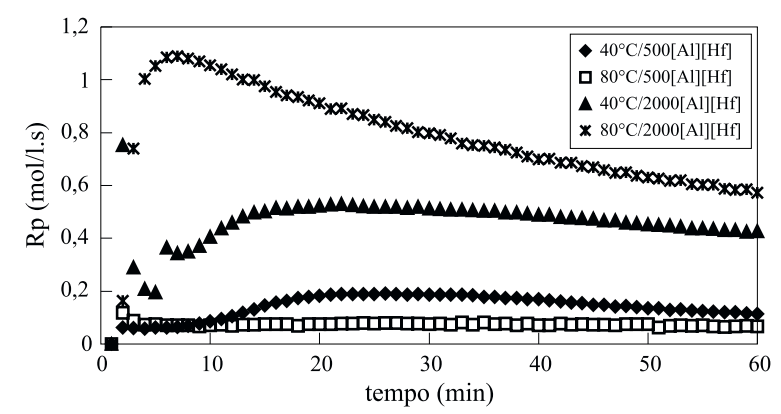

Figura 9. Perfil de velocidade de polimerização de propileno com o catalisador $\mathrm{Et}(\mathrm{Ind})_{2} \mathrm{HfCl}_{2}$

ponte de dimetilsilano, tanto com zircônio ou háfnio, foram em geral superiores às dos catalisadores com ponte de etilideno, assim como os pesos moleculares, a estereorregularidade e a temperatura de fusão dos polipropilenos sintetizados. Porém os teores de isotaticidade foram relativamente baixos comparando-se com os obtidos pelos sistemas Ziegler-Natta convencionais. É importante salientar que nenhuma fração de polímero atático foi obtida nos experimentos realizados, como seria esperado para o caso do emprego de catalisador Ziegler-Natta convencional. Isto significa que o grau de erro nas inserções monoméricas existe virtualmente em todas as cadeias poliméricas do produto obtido.

Analisando a estrutura dos dois catalisadores, observa-se que no complexo com ponte de dimetilsilano os ligantes possuem um ângulo de inclinação entre si superior ao do complexo com ponte etilidênica, que inclui dois átomos de carbono. Conseqüentemente, $o$ monômero tem um maior acesso ao centro ativo no catalisador com ponte de dimetilsilano e, portanto maior atividade catalítica é verificada. Maior peso molecular e estereorregularidade poderiam ser esperados pelos polímeros obtidos com o sistema com ponte de etilideno, porém a ponte $\mathrm{C} 2$ permite uma flexibilidade e mobilidade dos ligantes na molécula do complexo metalocênico, com a conseqüente interação do $\mathrm{H}-\beta$ da cadeia em crescimento com o sítio de coordenação e a inserção monomérica com estereoerros. Isso implica, respectivamente, na queda do peso molecular devido à reação de transferência de cadeia e na diminuição do teor de isotaticidade. Estereoerros na inserção monomérica provocam defeitos nos cristais do polímero, diminuindo assim sua temperatura de fusão.

Em relação a polidispersão, ela é baixa nos dois casos, permanecendo em valores próximos a 2 . 
Tabela 7. Modelos propostos e coeficiente de correlação (Ccor) para todas as variáveis dependentes estudadas

$\mathrm{SiMe}_{2}(\text { Ind })_{2} \mathrm{ZrCl}_{2}$

\begin{tabular}{|c|c|c|}
\hline Variável & modelo & Ccor \\
\hline atividade & $Y 1=3160 X_{1}+1790 X_{2}+1896\left(X_{1}\right)^{2}+1896\left(X_{2}\right)^{2}+1790 X_{1} X_{2}+10853$ & 0,986 \\
\hline atividade* & $Y 1 *=8852 X_{1}+3376 X_{2}+454\left(X_{1}\right)^{2}+454\left(X_{2}\right)^{2}+3377 X_{1} X_{2}+9520$ & 0,997 \\
\hline $\mathrm{Tm}$ & $Y 2=-9,25 X_{1}-2,75 X_{2}+2,29\left(X_{1}\right)_{2}+2,29\left(X_{2}\right)_{2}-3,25 X_{1} X_{2}+133,6$ & 0,997 \\
\hline Mw & $Y 3=-55180 X_{1}+2856 X_{2}+20993\left(X_{1}\right)^{2}+20993\left(X_{2}\right)^{2}-1067 X_{1} X_{2}+30574$ & 0,996 \\
\hline $\mathrm{Mw} / \mathrm{Mn}$ & $\mathrm{Y} 4=0,45 \mathrm{X}_{1}-0,05 \mathrm{X}_{2}-0,2583\left(\mathrm{X}_{1}\right)^{2}-0,2583\left(\mathrm{X}_{2}\right)^{2}-0,05 \mathrm{X}_{1} \mathrm{X}_{2}+2,76$ & 0,983 \\
\hline \multicolumn{3}{|l|}{$\operatorname{Et}(\operatorname{Ind})_{2} \mathrm{ZrCl}_{2}$} \\
\hline atividade & $\mathrm{Y} 1=1195 \mathrm{X}_{1}+1185 \mathrm{X}_{2}-2276\left(\mathrm{X}_{1}\right)^{2}-2276\left(\mathrm{X}_{2}\right)^{2}-555 \mathrm{X}_{1} \mathrm{X}_{2}+8387$ & 0,997 \\
\hline atividade* & $Y 1 *=4210 X_{1}+946 X_{2}-1038\left(X_{1}\right)^{2}-1038\left(X_{2}\right)^{2}-242 X_{1} X_{2}+7357$ & 0,997 \\
\hline $\mathrm{Tm}$ & $\mathrm{Y} 2=-0,97 \mathrm{X}_{1}-0,094 \mathrm{X}_{2}+113,0$ & 0,983 \\
\hline $\mathrm{Mw}$ & $Y 3=-241751 X_{1}-3175 X_{2}+7412\left(X_{1}\right)^{2}+7412\left(X_{2}\right)^{2}-1325 X_{1} X_{2}+19500$ & 0,999 \\
\hline $\mathrm{Mw} / \mathrm{Mn}$ & $Y 4=-0,15 X_{1}-0,05 X_{2}-0,125\left(X_{1}\right)^{2}-0,125\left(X_{2}\right)^{2}-0,05 X_{1} X_{2}+2,39$ & 0,999 \\
\hline$\%$ mmmm & $Y 5=-0,98 X_{1}+0,016 X_{2}+66,7$ & 0,984 \\
\hline \multicolumn{3}{|c|}{$\mathrm{SiMe}_{2}(\mathrm{Ind})_{2} \mathrm{HfCl}_{2}$} \\
\hline atividade & $Y 1=400 X_{1}+120 X_{2}+1592,7-206,3\left(X_{1}\right)^{2}-206,3\left(X_{2}\right)^{2}$ & 0,988 \\
\hline atividade* & $Y 1^{*}=1391 X_{1}+164 X_{2}+85 X_{1} X_{2}+1919-9\left(X_{1}\right)^{2}-9\left(X_{2}\right)^{2}$ & 0,997 \\
\hline $\mathrm{Tm}$ & $Y 2=-4,3 X_{1}+0,75 X_{2}+0,25 X_{1} X_{2}+135-2,4\left(X_{1}\right)^{2}-2,4\left(X_{2}\right)^{2}$ & 0,970 \\
\hline Mw & $\mathrm{Y} 3=17868+\exp \left(11,46-1,11 \mathrm{X}_{1}-0,13 \mathrm{X}_{2}\right)$ & 0,998 \\
\hline $\mathrm{Mw} / \mathrm{Mn}$ & $\mathrm{Y} 4=1,782788+\exp \left(-0,827-0,905 \mathrm{X}_{1}-0,042 \mathrm{X}_{2}\right)$ & 0,999 \\
\hline \multicolumn{3}{|l|}{$\mathbf{E t}(\operatorname{Ind})_{2} \mathbf{H f C l}_{2}$} \\
\hline atividade & $Y 1=50 X_{1}+690 X_{2}+200 X_{1} X_{2}+1780-420\left(X_{1}\right)^{2}-420\left(X_{2}\right)^{2}$ & 1,000 \\
\hline atividade* & $Y 1^{*}=740 \mathrm{X}_{1}+1087 \mathrm{X}_{2}+766 \mathrm{X}_{1} \mathrm{X}_{2}+2306-492\left(\mathrm{X}_{1}\right)^{2}-492\left(\mathrm{X}_{2}\right)^{2}$ & 0,970 \\
\hline $\mathrm{Tm}$ & $Y 2=-8,3 X_{1}-0,3 X_{2}-0,3 X_{1} X_{2}+129,3-1,8\left(X_{1}\right)^{2}-1,8\left(X_{2}\right)^{2}$ & 0,999 \\
\hline Mw & $Y 3=9712,98+\exp \left(10,85-1,29 X_{1}-0,117 X_{2}\right)$ & 0,999 \\
\hline $\mathrm{Mw} / \mathrm{Mn}$ & $\mathrm{Y} 4=1,802+\exp \left(-28,37+27,87 \mathrm{X}_{1}-0,47 \mathrm{X}_{2}\right)$ & 0,961 \\
\hline
\end{tabular}

*atividade específica; Tm- temperatura de fusão; Mw- peso molecular ponderal médio, Mw/Mn- polidispersão; mmmm- percentagem de pentades isotáticas; X1- temperatura de polimerização; X2- razão [A1]/[Metal de transição]; valores de X1 e X2 normalizados, conforme Tabela 2

\section{Conclusão}

De acordo com os resultados obtidos, pode-se concluir que os catalisadores a base de zircônio são os de maior atividade catalítica, enquanto que os hafnocenos produzem polipropileno com peso molecular mais elevado, conforme já esperado. Os complexos com ponte dimetilsilânica produziram polipropileno com maior peso molecular, estereorregularidade e, consequentemente, temperatura de fusão.
Para os quatro sistemas catalíticos estudados, conclui-se que o planejamento estatístico é uma ferramenta útil para se compreender o comportamento dos sistemas catalíticos metalocênicos. Porém, o acompanhamento da velocidade de polimerização com o tempo contribuiu fortemente para a análise dos dados obtidos.

\section{Referências Bibliográficas}

1. Scheirs, J. \& Kaminsky, W. - "Metallocene based 
Polyolefins", Vol II, John Wiley \& Sons Ltd, New York (2000).

2. Benedikt, G. M. \& Goodall, B. - "Metallocene Catalyzed Polymers-Materials, Properties, Processing \& Markets”, Plastics Design Library, New York (1998).

3. Fink, G.; Mulhaupt, R. \& Brintzinger, H.H. - "Ziegler Catalysts - Recent Scientific Innovations and Technological Improvements”, Springer-Verlag, New York (1995).

4. Kaminsky, W. - Catalysts Today, 62, p 23, (2000).

5. Razavi, A. - C. R. Acad. Sci. Paris, Série lic, Chemistry, p. 315 (2000)

6. Pal, K. - International Polymer Science and Technology, 26, p.57 (1999).

7. Mears, S. - "Polymers- structure and bulk properties", D. Van Nostrand Company, London (1967).

8. Leaversuch, R.D. - Modern Plastic International, 7, p.46 (1996).
9. Viville, P.; Daoust, D.; Jonas, A. M.; Nystem, B.; Legras, R.; Dupire, M.; Michel, J. \& Debras, G. - Polymer 42, p.1953 (2001).

10. Loos, J.; Buhk, M.; Petermann, J.; Zowmis, K. \& Kaminsky, W. - Polymer, 37, p. 387 (1996).

11. Ewen, J.A.; Jones, R.L.; Razavi, A. \& Ferrara, J. D. - J. Am Chem Soc. 110, p.6255, (1992).

12. Wild, F. R.W. P.; Zzsolnai, L.; Huttner, G. \& Brintzinger, H. H. J. - Organomet. Chem., 232, p.233 (1982).

13. Samuel, E. \& Rausch, M.D.J. - Am. Chem. Soc., 19, p.6263 (1973).

14. Bravakis, A. M.; Bailey, L. E.; Pigeon, M. \& Collins, S. - Macromolecules, 31, p.1000 (1998).

15. Box, G. E. P.; Hunter, W. G. \& Hunter, J. S. - "Statistics for experiments", John Wiley, New York (1978).

Recebido: 23/02/01

Aprovado: 22/03/02 